\title{
L'acrobate et le clown : notes sur la corporéité comique dans l'œuvre de Valère Novarina
}

Didier Plassard

\section{OpenEdition}

\section{Journals}

Édition électronique

URL : http://journals.openedition.org/recherchestravaux/302

DOI : 10.4000/recherchestravaux.302

ISSN : 1969-6434

Éditeur

UGA Éditions/Université Grenoble Alpes

\section{Édition imprimée}

Date de publication : 15 octobre 2006

Pagination : 91-95

ISBN : 978-2-84310-099-4

ISSN : 0151-1874

\section{Référence électronique}

Didier Plassard, « L'acrobate et le clown : notes sur la corporéité comique dans l'œuvre de Valère Novarina », Recherches \& Travaux [En ligne], 69 | 2006, mis en ligne le 18 décembre 2013, consulté le 08 septembre 2020. URL : http://journals.openedition.org/recherchestravaux/302 ; DOI : https:// doi.org/10.4000/recherchestravaux.302 


\section{L'acrobate et le clown: notes sur la corporéité comique dans l'œuvre de Valère Novarina}

Toute prière qui s'élève demande son ombre comique, sa contrefaçon.

V. NOVARINA

Au seuil des années 1970, alors que le théâtre français se trouve déchiré entre la génération de Jean Vilar et celle que fascine le Living Theatre, entre les partisans de l'accès du plus grand nombre aux institutions culturelles et ceux qui rêvent de créations collectives, anticipatrices d'une révolution à venir, la revue Travail théâtral publie, dans une indifférence à peu près générale, L'Atelier volant de Valère Novarina ${ }^{\mathrm{I}}$. Cette première pièce, qui met en scène de façon burlesque les relations d'un patron avec ses employés, porte assurément les marques de son temps, mais elle montre déjà un traitement singulier et puissamment comique du corps, aussi éloigné de l'abstraction beckettienne que des montages postbrechtiens ou des nouvelles dramaturgies réalistes qui vont s'imposer avec le théâtre du quotidien. Refaisant le trajet accompli par les avant-gardes des années 1920, l'écriture de Novarina emprunte au comique du cirque et des tréteaux forains : ainsi met-elle en jeu des personnages grotesques, multipliant les gags, les actions mécaniques et les déguisements pour représenter le monde du travail sous la forme d'un univers carnavalesque, critique et joyeux à la fois. L'aliénation créée par les rapports de production ne se traduit pas seulement dans les jeux clownesques des employés et du couple de patrons, Monsieur Boucot et Madame Bouche (lesquels rappellent irrésistiblement le Père et la Mère Ubu d'Alfred Jarry), mais elle affecte aussi la représentation du corps par et dans le langage, comme le montre par exemple la scène au cours de laquelle l'un des employés traverse une "crise de sentiments maternels»:

I. V. Novarina, «L'Atelier volant», dans Travail théatral, n V, Lausanne, La Cité, automne I97I, p. 46-87. 
LE PÈRE: Je suis assis au centre de mes meubles et tous mes objets viennent boire. Assiste, chère épouse, à la formation de mon deuxième corps... [...] Je nourris une nombreuse famille: femme, enfants et objets de ménage. Je donne. Je suis couvert d'une abondance de seins...

LA MÈRE: Mais tu n'as pas de lait, René!

LE PÈRE: Je fabrique de la monnaie et mes petits viennent me la manger. C'est pourquoi j'aimerais que l'on se souvienne de moi sous la forme d'une mère cochon.

LA MĖRE: René, tu délires! Tu n'as pas honte de dire ça?

LE PÈrE: Non. J'ai des seins, Marie, je te jure. Je suis couvert de seins.

LA MÈRE: D'où vient ton lait?

LE PÈRE: Il vient de là-bas où je travaille: je gagne soixante-quinze mille sur la croix... je ne sais trop comment. J'ai porté ma patience: toute ma vie, pour vous, je me suis sacrifié...!

LA MÈRE: Vous êtes fou, je vais vous battre, vous êtes une vraie femelle! Je ne veux plus de vous, jamais!

LE PÈRE: Ne frappez pas la vache à lait! (Elle lui saute au cou). ${ }^{2}$

L'œuvre théâtrale qui va suivre peut être partagée sommairement en trois périodes: jusqu'au milieu des années I980 environ, Novarina explore longuement une langue toujours plus tourmentée, travaillée par des déformations, des pérégrinismes et des néologismes, en même temps qu'il connaît les plus grandes difficultés pour faire jouer et même publier ses textes, Le Babil des classes dangereuses (1972, publié en 19783) ou La Lutte des morts (1974, publié en 1979). À partir du Drame de la vie (1984) et du Discours aux animaux (I987), le soutien d'une maison d'édition (P.O.L.), la rencontre d'interprètes comme André Marcon et la décision qu’il prend de mettre lui-même en scène ses œuvres, présentées notamment au festival d'Automne et au festival d'Avignon, conduisent l'écrivain à devenir avec Bernard-Marie Koltès l'une des figures emblématiques du retour des auteurs dans le paysage théâtral français. Si sa langue s'allège, si les structures dramaturgiques dont il use se clarifient, Novarina demeure fidèle cependant à une même ligne d'écriture, reconnaissable dans Vous qui habitez le temps (I989), Je suis (I99I) ou La Chair de l'homme (1995). Une troisième période, enfin, commence avec la rencontre de Claude Buchvald qui porte sur la scène successivement Vous qui habitez le temps en 1995, Le Repas (1996), L'Avant-dernier des hommes (1997) et L'Opérette imaginaire (1998), ouvrant aux ouvres de l'auteur la voie d'un très important succès public et d'une fortune critique unanime. Le Jardin de reconnaissance (1997), L'Origine rouge (2000) ou La Scène (2003), que Novarina met lui-même en scène, poursuivent le même mouvement d'ouverture et de variation autour

2. Ibid., p. 50 .

3. Toutes les œuvres théâtrales de Valère Novarina étant publiées aux éditions P.O.L. (Paris), je mentionnerai seulement la date de leur parution. 
de ce qui constitue le questionnement central de son œuvre : l'étrangeté, tout à la fois comique et terrifiante, du lien du corps et de la parole.

Je ne sais pas pourquoi la parole était faite mais certainement pas pour être un jour descendue dans les corps. Tout le scandale, toute la catastrophe vient de là : de la mise en chair de la parole. Elle nous est tombée par fatal accident. La Viande et le Verbe auraient dû vivre dans deux mondes séparés. ${ }^{4}$

Beaucoup plus qu'un répertoire de figures et de gestes parmi d'autres, l'idée d'un corps comique, chez Valère Novarina, apparaît en effet comme la définition même de la condition humaine, partagée entre les accidents de la chair et le souffle d'une parole venue d'ailleurs. Ainsi qu'il s'en explique dans ses essais ou dans ses entretiens, le travail même de l'acteur, en tant qu'il associe un corps et un texte étrangers l'un à l'autre, est pour lui l'exacte allégorie de notre humanité. Ses propres mises en scène, suivant en cela celles que réalise Claude Buchvald, donnent une première visibilité à ce conflit du verbe et de la matière: interprètes aux statures et aux silhouettes nettement différenciées, costumes aux coupes et aux proportions clownesques, immobilisation des corps dans des attitudes et des encadrements qui les transforment en pantins d'eux-mêmes. L'humanité qui se produit sur la scène des pièces de Valère Novarina est toujours puissamment typée, en une série de contrastes que l'auteur et metteur en scène, peintre lui-même, aime à rapprocher des manières de quelques grands maîtres de la peinture:

Dans Le Jardin de reconnaissance, Jean-Quentin Châtelain travaillait comme Soutine, Roséliane Goldstein comme Füssli et Wu Zhen, Agnès Sourdillon comme Miró. Laurence Mayor, je la compare à Chardin et à William Blake, Pascal Omhovère à Arnulf Rainer, Znyk à Picasso. Michel Baudinat joue comme Wölfli, André Marcon comme Masaccio, Cézanne. Paroles et gestes sont jetés, dépensés, matériels, livrés, spectaculaires, en face. L'acteur parle dans une lutte de couleurs. ${ }^{5}$

Plus encore, ce sont les clowns, les artistes de music-hall et les acteurs comiques de la scène ou de l'écran qui constituent selon lui non seulement des modèles d'interprétation, mais aussi et surtout les plus justes représentants de l'humain. Admirateur de Félix Mayol et de Grock, truffant ses œuvres de chansons qui sont autant de clins d'œil au répertoire des caf'conc' et des chanteurs populaires, Novarina consacre à Louis de Funès ses pages les plus enthousiastes, allant jusqu'à comparer son jeu à celui d'Hélène Weigel :

Je n'ai vu ces deux grands artistes sur scène qu'une fois: Funès dans Oscar et Weigel dans La Mère. [...]

Weigel excellait dans le "parlé-chanté»: Sprechgesang; Louis de Funès dans le «marché-dansé»: Schrittgetanz. Sa silhouette était celle d'un danseur exultant

4. V. Novarina, "Pour Louis de Funès", Le Théâtre des paroles, P.O.L., Paris, I989, p. I30.

5. V. Novarina, Devant la parole, P.O.L., I999, p. 64-65. 
ou soudainement d'un dépressif pétrifié. "Arrêté-bondi». Le grand maître des mimiques, des verbigérations muettes et des hurlements tus. Le cinéma ne conserve trop souvent qu'une image partielle de son art en n'en filmant que les crises aiguës: l'accès grimacier, les mille colères, le bondissement n'étaient au théâtre qu'un moment de son jeu, une intensité suprême, qu'il fallait savoir attendre, comme la danse du shité dans le nô, qu'après un long calme tendu et comme un couronnement de l'émotion. ${ }^{6}$

L'acteur emblématique du cinéma comique français des années I960 et I970 devient ainsi l'incarnation d'une quintessence de l'art du comédien et de l'humanité réunies, dans un double mouvement de déchirement des apparences et de métamorphoses incessantes.

Le visage de Louis de Funès, quand il jouait, m’a toujours semblé, dans sa lumineuse maniaquerie, sans aucune ombre et très exacte, comme la figure même de la transfiguration comique, qui fait la face humaine apparaître de plein feu dans une sorte de gloire déchirée. Car le visage de l'homme n'est pas un pot à tendre bêtement aux projecteurs et objectifs des photographes, mais une surface qui doit se déchirer, une face transfigurée et saisie par-dedans qui doit trembler en deux par une force qui le prend et le pousse hors d'ici. ${ }^{7}$

Acteur Nul et Parfait, toujours Louis de Funès entrait en néant, en niant et en tourbillonnant. Il savait qu'il avait la tête ouverte par la parole. [...] Que la parole n'est rien d'autre que la musique de la lumière qui se prononce en nous malgré nous et qu'elle vient de plus loin que nous. Louis de Funès disait: «Ce sont dans les corps comiques que les paroles ont chuté. ${ }^{8}$

Élevant l'interprète des Aventures de Rabbi Jacobà la stature d'un moderne Zarathoustra9, Valère Novarina en fait un double idéalisé de lui-même, un guide spirituel auquel il prête situations et propos entièrement imaginaires: tout à la fois prophète, gardien de musée, acteur et philosophe, porteur des mêmes interrogations et des mêmes convictions que l'auteur. Si son savoir est principalement un savoir du corps, le Louis de Funès de Novarina peut aussi se faire critique d'art et théologien:

Dans le rôle du suisse de la cathédrale de Strasbourg, devant le portail sud, Louis de Funès disait: «Superposez ici mentalement ces deux statues jumelles où le sculpteur anonyme a représenté: un, la Synagogue aveugle - deux, l'Église sourde. " ${ }^{10}$

Louis de Funès, quand il gardait Cristo morto de Mantegna, portait toujours dans sa casquette cette phrase de Tertullien, recopiée à la main: "Le fils de Dieu a été crucifié, je le crois car c'est absurde; le fils de Dieu est mort, je n'en doute pas car c'est inepte; enseveli, il est ressuscité, c'est certain parce que c'est impossible. ${ }^{I I}$

6. V. Novarina, «Pour Louis de Funès», loc. cit., p. II5.

7. Ibid., p. 135-136.

8. Ibid., p. I29.

9. Novarina dit souvent de son essai «Pour Louis de Funès» qu'il est «une sorte de Also sprach Louis de Funès".

IO. V. Novarina, Devant la parole, op. cit., p. I2I.

II. Ibid., p. 122. 
Sur la scène de ce théâtre qui, tel le drame liturgique médiéval ou l'auto sacramental du Siècle d'or, assume d'emblée une signification allégorique - celle d'une représentation figurée de notre destinée terrestre -, les personnages que crée Valère Novarina apparaissent dès lors comme d'innombrables déclinaisons de l'étonnement d'exister, de la perplexité d'habiter un corps, de la recherche trébuchante des pouvoirs du langage. Leurs noms mêmes désignent cette dimension symbolique: La Voix d'Ombre, le Bonhomme Nihil, La Personne Creuse, L'Acteur Fuyant Autrui, Le Personnage du Corps, les Antipersonnes I et 2... Accompagnant un processus de germination incessant, qui fait surgir 2587 personnages dans Le Drame de la vie et 3I7I dans La Chair de l'homme, le recours toujours plus marqué au comique des formes populaires charge ces figures presque abstraites d'une corporéité aux multiples accidents cruels et burlesques. Corps sciés en deux, cadavres ressuscitant pour chanter, duo de l'Homme Sang et d'Autrui, s'entresaignant en proférant que «l'Homme n'est pas bon ${ }^{\mathrm{I2}}$ ", ce jeu de pantins désaccordés mime joyeusement les défaites et les victoires de la pensée, les entrechoquements de lumière et d'obscurité au milieu desquels nous nous débattons.

La figure centrale de cette corporéité comique, en ce qu'elle manifeste brutalement les limites que le corps impose à l'esprit, est la chute. Chute réelle d'un employé grimpé en haut d'un mât, à la fin de La Fuite de Bouche ${ }^{\mathrm{i3}}$. Chute surtout métaphorique, évoquée en paroles plutôt que réalisée sur la scène, dans le reste de l'œuvre - ou bien encore métamorphosée en une liste de 88 figures qu'exécutent des voltigeurs de cirque dans La Chair de l'homme ${ }^{14}$, puisque la chute n'est que la conséquence de l'envol, son "ombre comique». Acrobate et clown, ou plutôt acrobate puis clown, telle est la double définition du corps selon Valère Novarina: non pas dans la perspective d'une dérision du sublime, d'un énième coup de pied de l'âne à la vie spirituelle, mais dans la conviction intime que le mouvement d'élévation et l'accident qui oblige à tomber sont les deux faces indissociables de notre incarnation.

Le théâtre est un lieu où l'homme va pour monter et tombe. Sa chute est une prière. Il y a, dans le rire accompagnant le don du corps qui s'effondre, un dépouillement de soi ; il y a une vraie sainteté du clown. L'acrobate qui chute, exécute la preuve comique de l'offrande de notre corps à l'espace. ${ }^{\text {IS }}$

I2. V. Novarina, L'Opérette imaginaire, P.O.L., 1998, p. 87.

13. Voir V. Novarina, La Fuite de Bouche, Marseille, Jeanne Laffitte, 1978, p. II7. La Fuite de Bouche est une réécriture de L'Atelier volant destinée à une mise en scène de Marcel Maréchal.

I4. V. Novarina, La Chair de l'homme, Paris, P.O.L., I995, p. 439-443.

I5. V. Novarina, Devant la parole, op. cit., p. 83. 